\title{
Cephalopods Inspired Rapid Self-Healing Nanoclay Composite Coatings with Oxygen Barrier and Super-Bubble-Phobic Properties
}

\author{
Kengo Manabe, ${ }^{\dagger} *$ Emiko Koyama, ${ }^{\dagger}$ and Yasuo Norikane ${ }^{\dagger}$ \\ ${ }^{\dagger}$ Research Institute for Advanced Electronics and Photonics, National Institute of Advanced Industrial Science \\ and Technology (AIST), Higashi 1-1-1, Tsukuba, Ibaraki, 305-8565, Japan
}

KEYWORDS: biomimetics; cephalopod; self-healing; layer-by-layer; gas barrier

\begin{abstract}
Polymeric coatings with oxygen barrier properties are an important technology in food packaging that can extend the shelf life of food products and reduce waste. Although a typical technology in practical use is the deposition of metal or inorganic materials between multilayer films to reduce the oxygen transmission rate, once the film is damaged, oxygen permeates through the damaged area, damaging the packaged food. In addition, nanobrick wall structures consisting of nanoplatelet bricks have the potential to replace barrier films made of inorganic materials, however, they similarly lack repair performance or have slow repair speed despite having repair performance. Inspired by the rapid self-repair mechanism of cephalopods, the study develops a nanoclay-containing coating that can rapidly repair surface damage via water. By introducing $\mathrm{CaCl}_{2}$-derived counterions and montmorillonite for nanobrick wall structures into polyelectrolyte multilayers stacked by layer-by-layer self-assembly, the non-covalent polymer network is increased, resulting in mimicking a strong cephalopod-derived $\beta$-sheet structure and non-covalent intermolecular interactions derived from cephalopods. Regardless of the amount of montmorillonite added, the self-healing process was completed within $10 \mathrm{sec}$. The high-water retention at the surface showed super-bubble-phobicity in water and inhibited gas permeation. The oxygen permeability of the coatings with more than a certain amount of montmorillonite was less than $1 / 100$ of that of bare polyethylene. The ultra-fast self-healing gas barrier coating has the potential to be used not only for food products but also for electronics and pharmaceutical packaging and gas separation applications. The key technology developed in this study provides novel insights into the construction of self-healing membranes made of composite materials and will contribute to the formation of a sustainable society.
\end{abstract}

\section{INTRODUCTION}

Oxygen barrier thin films and coatings for packaging applications are of great interest for the stable protection of food, pharmaceuticals, and electronic devices..$^{1-3}$ In particular, in food packaging, it is an important technology to reduce oxygen permeability, which can inhibit food deterioration and consequently lead to food waste. ${ }^{4,5}$ Conventional packaging technology consists of building a multi-layered structure using lamination, vapor deposition, and other methods. ${ }^{6}$ In this multilayer structure, metals, metal oxides, and other inorganic materials are inserted between the plastic films as gas barrier layers. However, in recent years, various points have become apparent from both health and environmental perspectives, such as their leaching into food and problems related to separation at the time of disposal. ${ }^{7,8}$ In addition, as a fundamental issue, if the surface of the package is damaged during the transportation process or the display process at the store, the oxygen will flow into the package starting from the damaged part, and the deterioration of the content food will be significantly advanced. ${ }^{9}$

To overcome these limitations, a nanobrick wall structure has been devised using the layer-by-layer (LbL) self-assembly method to stack layered materials and polymers with nanoplatelets. ${ }^{10-12}$ Montmorillonite (MMT), which are clay materials (nanoclay), layered double hydroxide (LDH), and graphene are often used as nanoplatelets for thin films with gas barrier properties. ${ }^{13-15}$ MMT, in particular, is a good candidate for generalpurpose food packaging applications because of its low cost and ease of handling due to its high dispersibility in polymer solutions. The composite of the nanoclay and polymer exhibits remarkable gas barrier performance because of the tortuous pathway of gas molecules moving around the nanoplatelets, called the maze effect when oxygen permeates. ${ }^{16-18}$ Therefore, it has been shown that increasing the amount and orientation of the nanoplatelets can produce a low oxygen transmission rate (OTR) comparable to that of conventional inorganic thin films. Therefore, the LbL method is an effective construction method for both sides due to the advantage that the nanoplatelets can be oriented perpendicular to the oxygen diffusion direction and the amount of additives can be easily controlled by the initial concentration of the polymer solution. ${ }^{19-22}$

Another advantage of using LbL self-assembly is its selfhealing function. ${ }^{23,24}$ Polymer multilayers composed of weakly electrolyte polycations and polyanions can self-repair by watermediated membrane expansion and subsequent reformation of bonds between polymers. ${ }^{25,26}$ Polymer multilayers with selfhealing behavior have been investigated for various applications, and their application to antifouling, antibacterial, 
superhydrophobic, conductive, flame-retardant, and gas barrier membranes has been reported so far. ${ }^{27-31}$ Despite considerable efforts, however, self-healing using polymer multilayers is still slow in its repairing speed, taking more than 5 minutes at the minimum as a recent excellent example. ${ }^{32}$

Therefore, the purpose of this study is to develop multilayer coatings with quick repair speed while compositing nanoclay to the polymer multilayer for gas barrier performance. The concept for improving the self-repair speed was inspired by the self-repair mechanism of cephalopods such as squid (Figure 1). Previous studies have shown that cephalopods can self-repair their legs and other parts of their body. ${ }^{33-35}$ Their polypeptides are composed of a network of proteins and strong $\beta$-sheet nanostructures, which can be rapidly repaired upon damage by non-covalent intermolecular interactions and chain diffusion via hydrogen bonds. We focused on the bonding/non-bonding parts and hypothesized that doping of MMT and $\mathrm{CaCl}_{2}$ ions could suppress the bonding between polymers and realize quick self-repair. By ion doping only the polymer solution during coating construction and removing residual salts by rinsing with pure water, it is expected that the polymer chains can be controlled by counterion binding. ${ }^{36,37}$ In addition, the introduction of counterions can reduce the charge density of the polymer and change the conformation of the polymer from a loop structure to a train structure, which leads to improved water absorption and retention performance. ${ }^{38,39}$ Furthermore, since each nanoclay, MMT, is an independent platelet, it is expected not to interfere with the quick movement of the polymer. ${ }^{40}$ Therefore, the nanoclay and ion doping could enhance the mobility of the polymer network, resulting in the possibility of achieving rapid repair performance even before and after the introduction of the nanoclay.

Here, we introduced nanoclay at different concentrations into ion-doped multilayer membranes composed of a weak polyelectrolyte, branched polyethyleneimine (bPEI)/polyacrylic acid (PAA), and the results showed a repair time of 10 seconds. Moreover, independent of the concentration of the clay, it showed a quicker healing performance than the existing watermediated self-healing membranes. By increasing the concentration of nanoclay, a barrier film was constructed with an OTR of less than 0.1, the detection limit of the instrument, and an OTR of $1 / 100$ before and after coating. Furthermore, the high-water retention at the surface demonstrated super-bubble-phobicity and inhibited gas-bubble permeation in water. The results of this study are advantageous in that the repair speed is much faster than that of conventional self-healing gas barrier films, and the low cost of the mechanism can be expressed only by introducing electrolyte salts and nanoclay. In the future, it is expected that the self-healing film can be used to quickly repair defective parts of packages by using the moisture of the food contents or to reseal packages once they have been opened. Thin-film coatings with rapid self-healing performance will protect and extend the life of food products, thereby contributing economically and socially to the reduction of waste and the achievement of our Sustainable Development Goals (SDGs).

\section{- RESULTS AND DISCUSSION}

Water retention properties. LbL self-assembly is a powerful approach to the preparation of polymeric thin coatings, and the advantage is the ability to control coating thickness ranging from nano- to microscale and freely combine any substance, that can be dispersed in a solution, into the polyelectrolyte multilayer as an additive. ${ }^{41,42}$ To understand the impact of MMT nanoclay, the number of bilayers was unified to 30-bilayer for all coatings, and the $\mathrm{pH}$ of each solution was unadjusted by additional acid/base. The actual coating thickness slightly varied depending on the concentration of MMT (Figure 2a). Compared to the film thickness of $24.2 \mu \mathrm{m}$ without MMT, the film thickness slightly decreased to $23.3 \mu \mathrm{m}$ when $0.02 \mathrm{mg} / \mathrm{mL}$ of MMT was added, which was within the error range considering the standard deviation. When the concentration of MMT was increased to $0.2 \mathrm{mg} / \mathrm{mL}$, the film thickness increased to $26.8 \mu \mathrm{m}$, and when MMT was further added to $2 \mathrm{mg} / \mathrm{mL}$, the film thickness conversely decreased. It has been shown in previous a

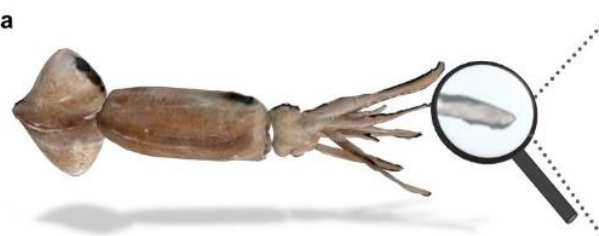

b

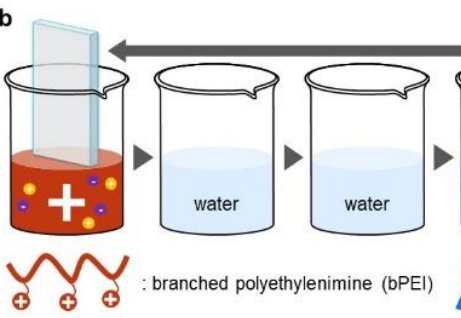

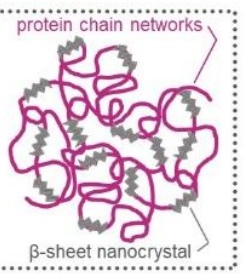

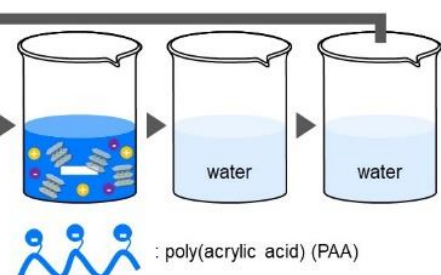

montmorillonite (MMT) c

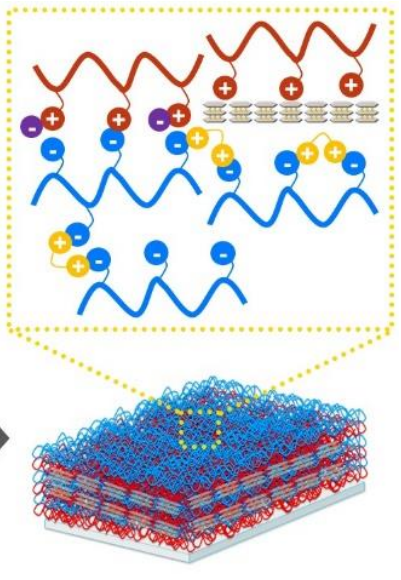

Figure 1. Design of cephalopods-inspired rapid self-healing nanoclay composite coatings. a Focus on a squid and the nanostructure of $\beta$-sheet nanocrystal and protein chain networks derived from the cephalopods' polypeptides. b Fabrication methodology for rapid self-healing nanoclay composite coatings via layer-by-layer self-assembly. c Completely deposited polymer multilayer on substrates. Ion-doped polyelectrolyte matrix containing nanoclay (MMT), either breaking a polycation/polyanion pair and acting as counterions. 

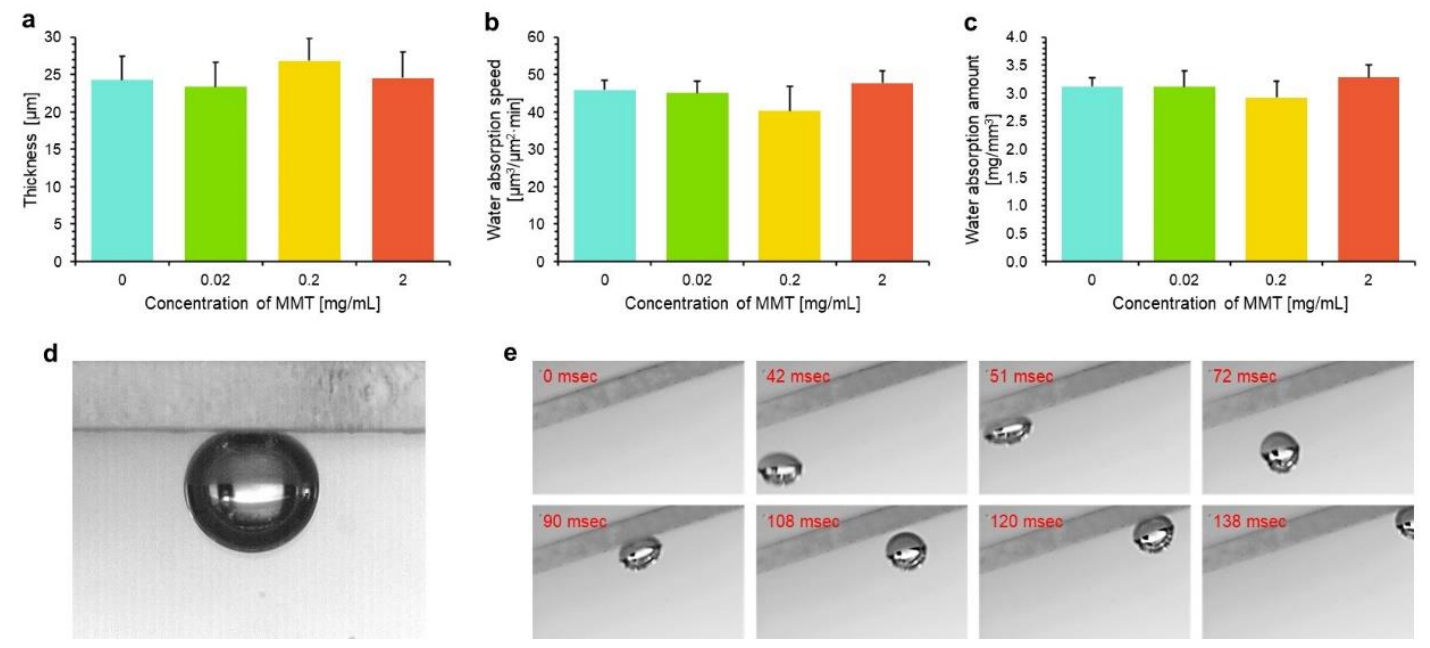

Figure 2. Water retention on self-healing nanoclay coatings. a Coating thickness, b water absorption rate, and $\mathbf{c}$ initial moisture content per unit volume of self-healing coatings with different concentration of MMT. d Static and e dynamic super-bubble-phobicity on the self-healing nanoclay coating with MMT $2 \mathrm{mg} / \mathrm{mL}$ for repelling and jumping gas bubbles under water, respectively.

studies that increasing the concentration of electrolyte salts inhibits the growth of the electrolyte membranes due to the increased charge shielding of the polymers in each component solution, and the additive MMT was expected to have the same effect. ${ }^{43}$ On the other hand, it has also been experimentally shown that as the immersion time in the MMT solution increases, the film thickness increases dramatically due to the increased frequency of MMT nanoplatelet migration relative to the surface. ${ }^{44}$ The question to be considered is whether MMT acts on the polymer in solution or on the surface deposition, with the concentration determining which is dominant. Therefore, the film thickness of the self-healing nanoclay coating could be attributed to the predominant effect of MMT on the surface deposition up to $0.2 \mathrm{mg} / \mathrm{mL}$, which promoted the film thickness growth, while at $2 \mathrm{mg} / \mathrm{mL}$, MMT affected the polymer network and inhibited the film thickness growth. The water uptake and water retention per minute of the prepared coatings were evaluated (Figure 2b, 2c). Increasing the concentration of MMT slightly affected the water absorption rate, with a decreasing trend up to an increase of $0.2 \mathrm{mg} / \mathrm{mL}$ of MMT and a
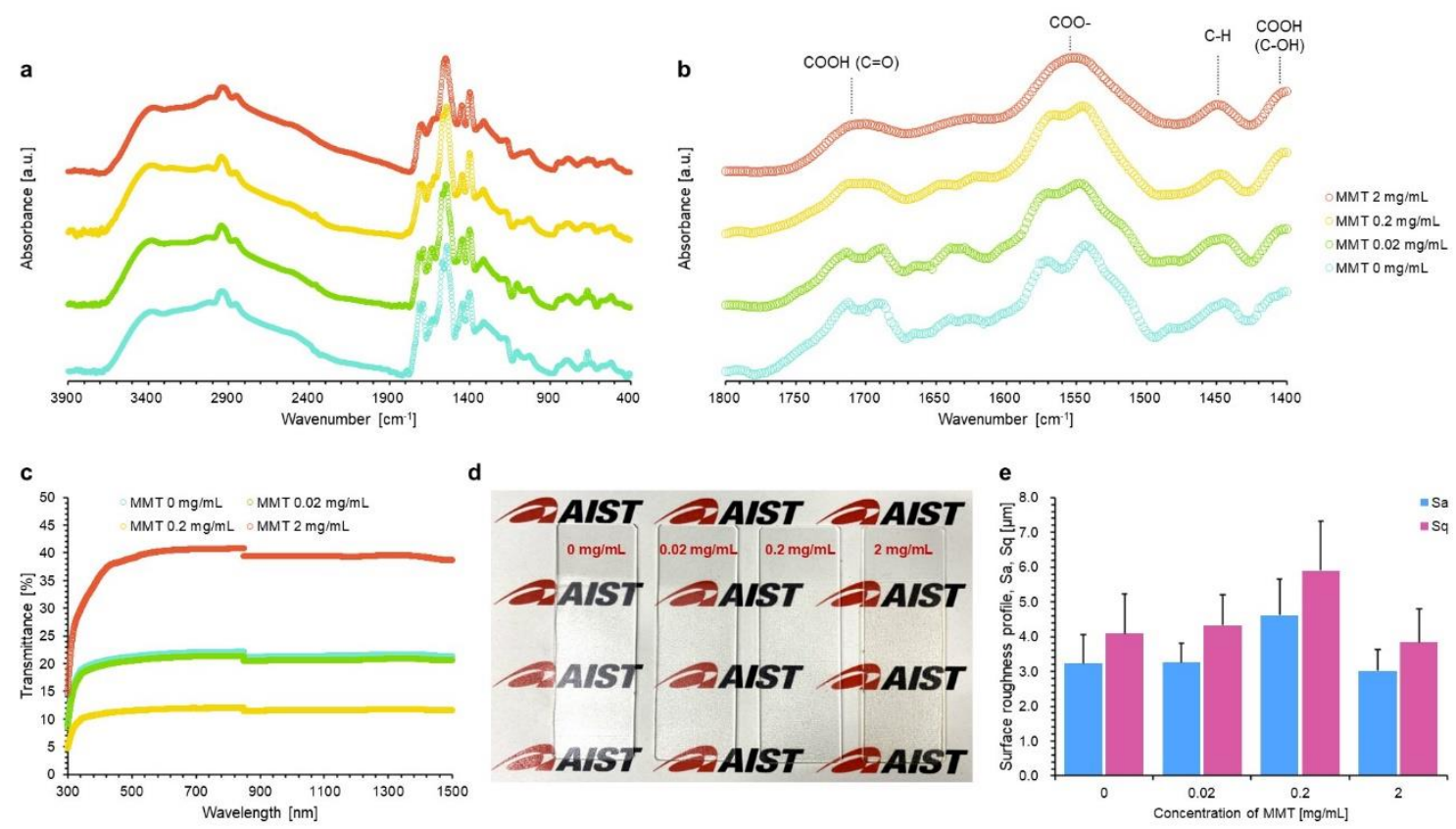

Figure 3. Chemical bonding analysis and optical features. a Wide-band and b narrow-band Fourier transform infrared (FT-IR) spectra of coatings with MMT 0 (sky), 0.02 (light green), 0.2 (yellow), and $2 \mathrm{mg} / \mathrm{mL}$ (red), respectively. c Measured transmittance changes of the coatings on glass substrates with different MMT concentration. d Photo image of each coating on glass substrates. e Surface roughness profiles, Sa (arithmetical mean height, light blue bars), and Sq (root mean square height, RMS, pink bars), of the coatings with different MMT concentration. 
return to the original water absorption rate at $2 \mathrm{mg} / \mathrm{mL}$. The result was evaluated from the contact area and height of the water droplets on the surface as an experimental procedure. Therefore, if the surface roughness is large, air pockets may be created between the water droplet and the surface in the initial state. The phenomenon would have affected the deviation, resulting in a decrease in the water absorption rate and a larger variation. On the other hand, when the water retention per unit volume was evaluated in the membranes after immersion in pure water, the effect of the concentration of MMT was found to be small. This indicates that the complete contact of water with the surface can absorb and retain the same amount of water, independent of the addition of MMT.

An application of the high-water holding capacity at the surface is the super-bubble-phobic property in water. When the bubbles were placed on the surface, the contact angle was more than 150 degrees, which completely inhibited the adhesion of gases (Figure 2d). The bubbles released toward the surface and hitting the coating were observed with a high-speed camera (Figure 2e and Movie S1). The highly water-holding surface formed a stable water film on the top surface as it absorbed water from the water bath, repelling the colliding bubbles and preventing them from sticking. As a result, the self-healing nanoclay coating in this study has the potential to be applied to inhibit gas permeation in liquids.

Chemical and optical analysis. In ordinary polymer multilayers by LbL, polycations and polyanions are constructed by depositing them by strong electrostatic bonds. In the present work, ion doping and addition of MMT nanoclay are carried out to mimic the strong $\beta$-sheet and non-covalent polymer network derived from cephalopods, and the effect is verified (Figure $3 \mathrm{a}$ and $3 b$ ). In comparison with the FTIR spectra of bulk bPEI and PAA in the previous study, no new peaks were formed by the addition of ion doping and MMT nanoclay. ${ }^{45}$ In conventional non-ion-doped polymer multilayers, the carboxyl group of PAA completely dissociates and bonds with bPEI, resulting in the appearance of a strong COO-peak $\left(1560 \mathrm{~cm}^{-1}\right)$ and no COOH peak $\left(\sim 1714 \mathrm{~cm}^{-1}\right)$ (Figure S1). On the other hand, the addition of ion doping and MMT in the present study resulted in a large and clear appearance of the $\mathrm{COOH}$ peak $\left(\sim 1714 \mathrm{~cm}^{-1}\right)$ in addition to the COO- peak $\left(1560 \mathrm{~cm}^{-1}\right)$. Hence, the ion doping and nanoclay were shown to affect the functional groups of each polymer and inhibit the bonding between polycation and polyanion.

It was also found that the peaks associated with carboxyl groups were changed by increasing the amount of MMT added. A doublet centered on the original $\mathrm{COOH}$ peak was observed within the $\mathrm{COOH}$ peak, and the increase in MMT changed this doublet peak into a single peak. The doublet peak is attributed to the intermolecular and intramolecular hydrogen bonds originating from the $\mathrm{COOH}$ of PAA. ${ }^{46}$ Hence, the ion doping suppressed the dissociation of the carboxyl groups and the hydrogen bonding appeared stronger, while the addition of MMT suppressed the hydrogen bonding, suggesting that MMT was deposited on the bPEI along with PAA. This result is consistent with the previous discussion on film thickness, where the increase in MMT inhibits the network between and within polymer molecules, thereby suppressing the increase in film thickness.

The optical effects of the addition of MMT were evaluated (Figure $3 \mathrm{c}$ and $3 \mathrm{~d}$ ). When the coating was deposited on a glass substrate, the transmittance remained constant at about $20 \%$ before the addition of MMT (note: the sharp absorption around $300 \mathrm{~nm}$ wavelength is due to the glass substrate). The transmittance of the coating with $0.02 \mathrm{mg} / \mathrm{mL}$ of MMT was slightly reduced relative to the coating before addition. When $0.2 \mathrm{mg} / \mathrm{mL}$ of MMT was added, the transmittance decreased further, showing a transmittance of about $10 \%$. The overall transmittance of the coating with $2 \mathrm{mg} / \mathrm{mL}$ of MMT increased, but as shown in the transmittance curve and photographs, the decrease in transmittance at wavelengths between 300 and $500 \mathrm{~nm}$ versus wavelengths above $500 \mathrm{~nm}$ was larger than that of the coating under other conditions, and the entire coating turned slightly yellow in the photographs. It is suggested that the film thickness, refractive index, and surface roughness have a combined effect on the transmittance variation. The film thickness was maximized at $0.2 \mathrm{mg} / \mathrm{mL}$, as mentioned earlier, which increased the optical path length for vertically incident light to hit the scattering medium, resulting in increased scattering. According to the

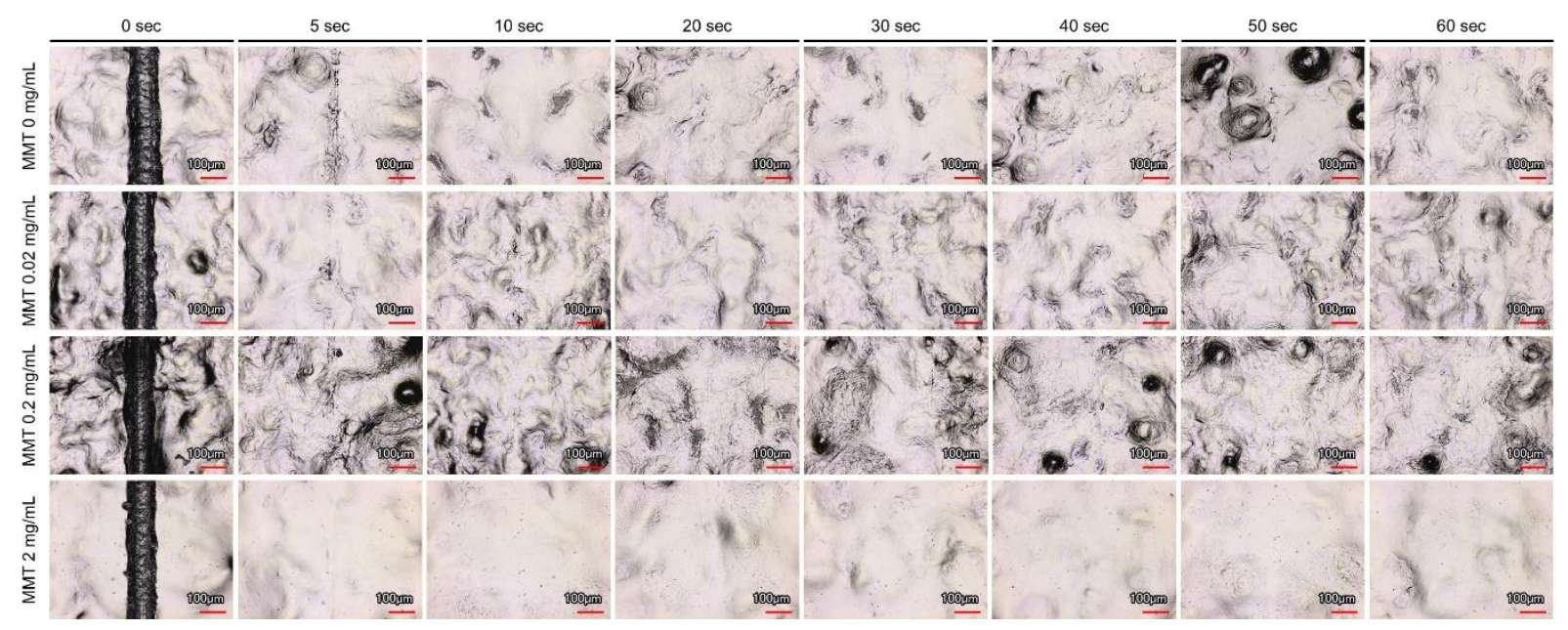

Figure 4. Self-healing observations. Surface images of the self-healing nanoclay coatings at different exposure time in water with different MMT concentrations, $0 \mathrm{mg} / \mathrm{mL}$ (first line), $0.02 \mathrm{mg} / \mathrm{mL}$ (second line), $0.2 \mathrm{mg} / \mathrm{mL}$ (third line), and $2 \mathrm{mg} / \mathrm{mL}$ (fourth line), were observed using a 3D laser scanning microscopy at different positions. The red scale bar is $100 \mu \mathrm{m}$. 


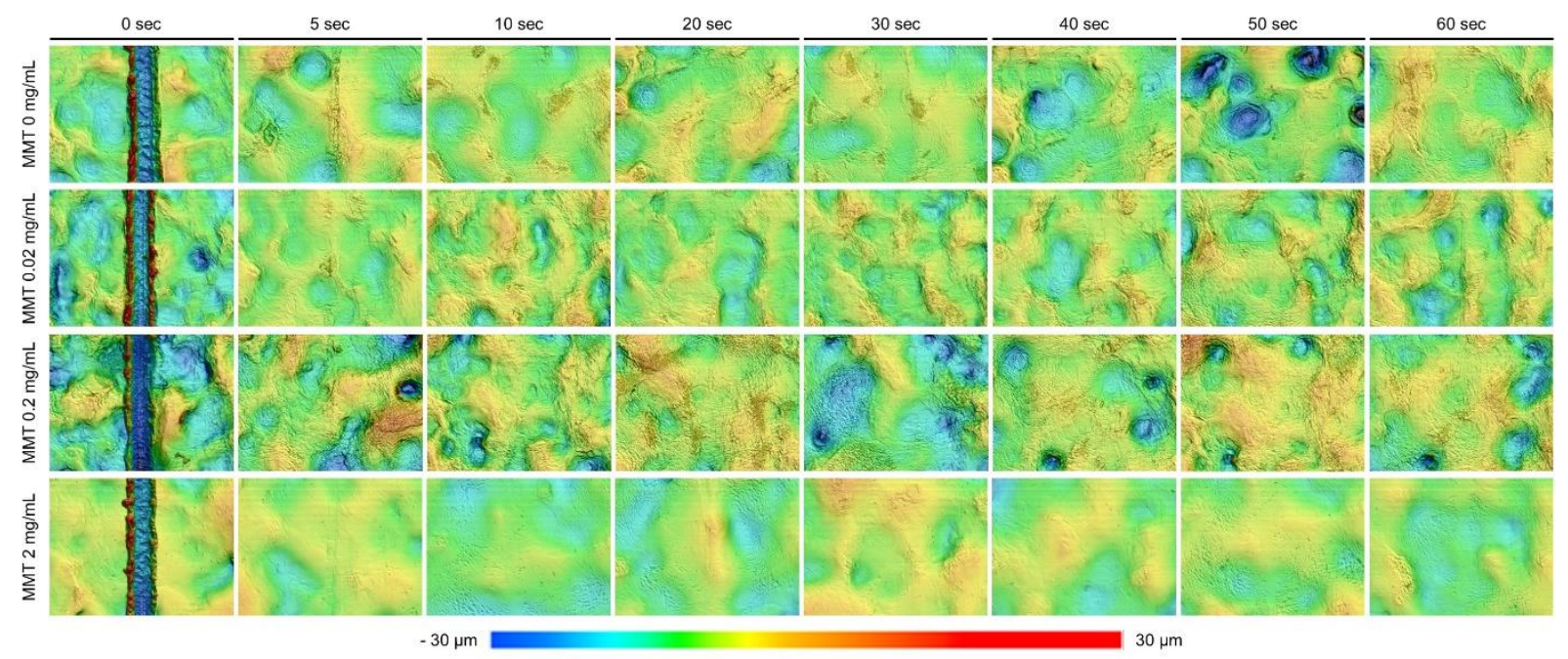

Figure 5. Height analysis of self-healing coatings. 3D height images of the corresponding self-healing nanoclay coatings in Figure 4 at different water-exposure time with MMT $0 \mathrm{mg} / \mathrm{mL}$ (first line), $0.02 \mathrm{mg} / \mathrm{mL}$ (second line), $0.2 \mathrm{mg} / \mathrm{mL}$ (third line), and $2 \mathrm{mg} / \mathrm{mL}$ (fourth line), were analyzed using a 3D laser scanning microscopy at different positions. The roughness scale bar is $-30-30 \mu \mathrm{m}$.

Fresnel equations, the refractive index of an $\mathrm{LbL}$ coating can be estimated using a simple mixing rule: ${ }^{47,48}$

$n_{l}=f_{\text {air }} n_{\text {air }}+f_{\text {polyecation }} n_{\text {polycation }}+f_{\text {polyanion }} n_{\text {polyanion }}+$

$f_{\text {additive }} n_{\text {additive }}$

where $f_{x}$ is the volume fraction, and $n_{x}$ is the refractive index of component $x$. The decrease in refractive index leads to a decrease in the surface scattering of the film. In the present coating, the refractive indices of bPEI, PAA, and MMT are 1.529, 1.527 , and 1.426, respectively. Although optical refractive index evaluation by ellipsometry could not be carried out in this study due to the low overall transmittance of the coating, the increase in the content of MMT would directly contribute to the decrease in the overall refractive index of the coating. The surface roughness was also evaluated (Figures 3e and S2). According to the results, Sa (arithmetical mean height), Sq (root mean square height, RMS), and Sz (maximum height) all increased with increasing MMT concentration up to $0.2 \mathrm{mg} / \mathrm{mL}$. In the coating with $2 \mathrm{mg} / \mathrm{mL}$ of MMT, Sa and Sq were decreased. Furthermore, $\mathrm{Sz}$ was found to be smaller than that of the coating before the addition of MMT. As for the surface roughness, the surface observation clearly showed the difference between the
MMT concentration conditions (Figure 4 and Figure 5). This suggests that the surface roughness that contributes to scattering may be suppressed by the excessive introduction of MMT, which led to the increase in transmittance.

Rapid self-healing performance. The surface repair was observed for different immersion times in water (Figure 4 and Figure 5). The repair performance was much faster than that of conventional polymer multilayers, and the repair of scratches of about $100 \mu \mathrm{m}$ was completed in about 10 seconds under all MMT concentration conditions, and the scratches on the surface were almost invisible. In terms of the height evaluation results, no significant scratches were observed after 5 seconds of immersion in water, but indentations were still observed on the surface. Later, when the immersion time was 10 seconds, no scratches could be discerned from the height evaluation. Even after further immersion in water, the damaged area was not exposed again, and the scratches and indentations disappeared completely. Interestingly, the concentration of MMT nanoclay added did not affect on the repair time. This result indicated that MMT did not inhibit the quick migration of macromolecules due to their independent nanoplatelet structures (i.e., broken nanostructures). The positive impact of independent nanoplatelet structures on gas barrier performance has been shown in
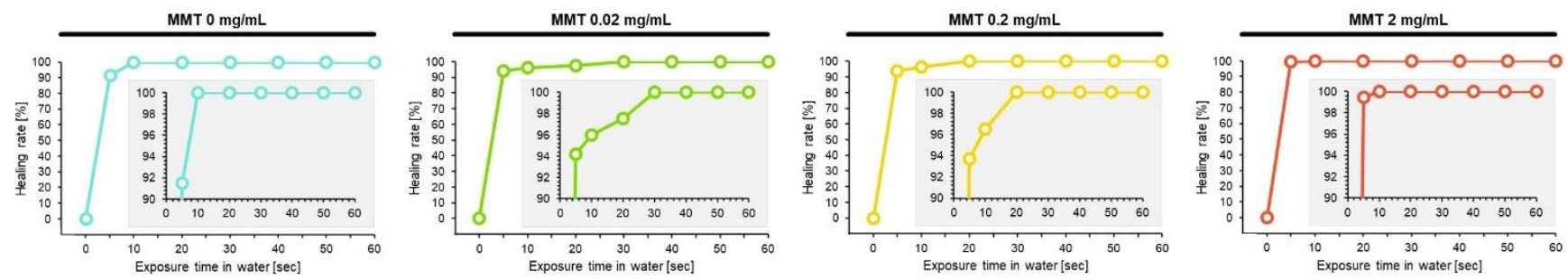

Figure 6. Rapid self-healing performance. Healing rate calculated using height difference in the level before and after exposure in water at different exposure time with different MMT concentrations, 0 (sky), 0.02 (light green), 0.2 (yellow), and $2 \mathrm{mg} / \mathrm{mL}$ (red), respectively. 
previous studies, and similarly, the properties were found to be valid for fast self-healing surfaces.

In multilayers constructed by the LbL self-assembly method, the polymer closer to the substrate expands predominantly, so that the substrate exposed by damage quickly becomes invisible. ${ }^{49}$ Therefore, the height of the damaged area was evaluated graphically as the self-healing performance to consider the selfhealing process to be complete when even the dent is completely erased, instead of the damage recovery in the lateral direction to the substrate (Figure 6 and Figure S3). The healing rate was calculated using the following formula: healing rate $[\%]=[\{$ height of reference surface (average height of coating excluding scar (dents) - average height in scar (dents) $\}$ / height of reference surface (average height of coating excluding scar $($ dents $))] \times 100$. From the detailed height evaluation, it was clear that more than $90 \%$ of the surface scratches were repaired after 5 seconds of water immersion for all MMT concentration conditions. The time to complete repair was $10,30,20$, and $10 \mathrm{sec}-$ onds at MMT $0,0.02,0.2$, and $2 \mathrm{mg} / \mathrm{mL}$, respectively. It should be noted that this calculation result does not take into account the roughness, so if the entire coating is uneven after deposition, the roughness will also be averaged together. However, it took less than 30 seconds to completely repair the coating under all conditions, suggesting that this is the fastest level of repair time for a polymer multilayer coating to recover surface scratches. From the above results on the repair performance, the addition of ion dope and MMT nanoclay enhanced the mobility of the polymer on the substrate by inhibiting the bonding between the polymers in the polymer multilayer, and achieved a quick selfhealing that could erase the surface scratches in the fastest 10 seconds.

Oxygen barrier performance of nanoclay coating. The nanobrick wall structure can reduce the oxygen transmission rate (OTR) by creating an extremely tortuous pathway for the permeating molecules due to the maze effect. The environmental humidity during the measurement was set to $0 \%$ and $50 \%$, and the OTR was measured (Figure 7). $0 \%$ humidity is a setting often used in general basic research tests, while 50\% humidity was used as an ISO-compliant condition. Polyethylene, which is often used in food packaging, was chosen as the base material. For polyethylene without coating, the OTR was 12900 and 8600 $\mathrm{mL} /\left(\mathrm{m}^{2} \cdot 24 \mathrm{~h} \cdot \mathrm{atm}\right)$ at 0 and $50 \%$ humidity, respectively, indicating a very high oxygen permeation. When a coating was applied to the polyethylene, the OTR was dramatically improved. First, in tests under $0 \%$ humidity conditions, the OTR was $1 / 10$ of the original OTR for all coatings, reducing the oxygen transmission rate. When the humidity was $0 \%$, the effect of the added concentration of MMT was small because the polymer multilayer film did not expand. It was also observed that the present polymer multilayer film cracked at $0 \%$ humidity (Figure S4). The polymer multilayer could not withstand the rapid decrease in humidity during the test, and this crack was the starting point for oxygen permeation, which resulted in a minimum OTR of $1000 \mathrm{~mL} /\left(\mathrm{m}^{2} \cdot 24 \mathrm{~h} \cdot \mathrm{atm}\right)$. On the other hand, under the condition of $50 \%$ humidity, the difference depending on the concentration of MMT added became larger. Under humid conditions, the polymer multilayer film may expand, and there is a risk of oxygen permeation through it. Without the addition of MMT, the OTR was reduced by half compared to that before coating. In the coating with $0.02 \mathrm{mg} / \mathrm{mL}$ of MMT, the OTR was slightly lower than before the addition. At $0.2 \mathrm{mg} / \mathrm{mL}$ of MMT, the OTR decreased sharply and showed $500 \mathrm{~mL} /\left(\mathrm{m}^{2} \cdot 24 \mathrm{~h} \cdot \mathrm{atm}\right)$. The MMT concentration was further increased and the coating with 2 $\mathrm{mg} / \mathrm{mL}$ added resulted in an OTR below the lower limit of the OTR evaluation system $(>0.1)$. Considering that the OTR decreased to $1 / 6$ with the increase in MMT concentration from $0.02 \mathrm{mg} / \mathrm{mL}$ to $0.2 \mathrm{mg} / \mathrm{mL}$, the value of $2 \mathrm{mg} / \mathrm{mL}$ is estimated to be less than 0.08 (less than $1 / 6$ of $500 \mathrm{~mL} /\left(\mathrm{m}^{2} \cdot 24 \mathrm{~h} \cdot \mathrm{atm}\right)$ ) even if we underestimated the value. Hence, compared to the case without coating, the restorative nanoclay coating was evaluated to have demonstrated oxygen barrier performance, suggesting that it was effective in reducing the OTR to $1 / 100$. It was also found that by increasing the concentration of MMT, the shielding effect of nanoplatelets of MMT was more dominant than the highway function of increasing oxygen permeability due to the expansion of polymer multilayer under high humidity.

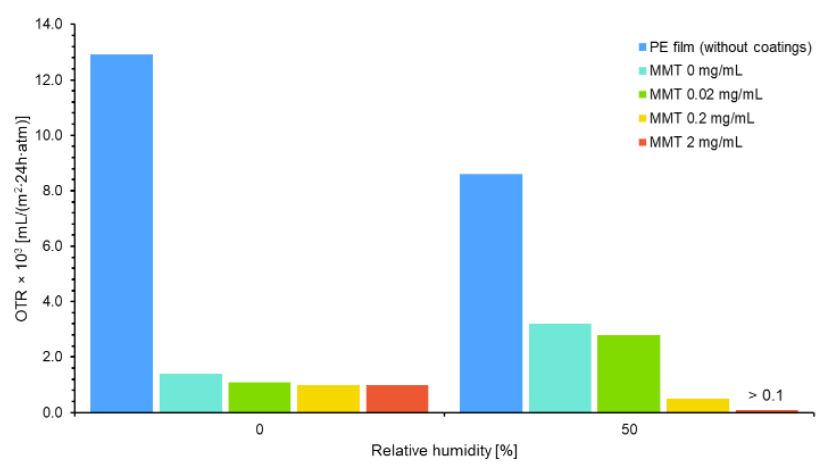

Figure 7. Oxygen transmission rate. Oxygen transmission rate (OTR) of a bare polyethylene (PE) film (without coatings, light blue bars) and self-healing nanoclay coatings with different MMT concentrations, 0 (sky bars), 0.02 (light green bars), 0.2 (yellow bars), and $2 \mathrm{mg} / \mathrm{mL}$ (red bars), at different relative humidity, respectively. The detection limit of the instrument is OTR $0.1 \times 10^{3} \mathrm{~mL} /\left(\mathrm{m}^{2} \cdot 24 \mathrm{~h} \cdot \mathrm{atm}\right)$.

\section{CONCLUSIONS}

By mimicking the self-healing mechanism of cephalopods through ion doping and nanoclay complexation, we have achieved a coating that can self-heal surface scratches at a very fast rate through water. The introduction of ions and nanoclay interfered with the strong electrostatic bonds between the polymers, improving the mobility of the polymer chain and completely repairing the surface in 10 seconds. The separate platelets of nanoclay did not disturb the mobility of the polymers and proposed a high repair performance independent of the MMT concentration. In the coating with $2 \mathrm{mg} / \mathrm{mL}$ of MMT, the nanobrick wall structure of the stacked platelets resulted in a reduction of gas permeation, and the oxygen permeability of the coating was less than 1/100 compared to that before coating, resulting in its effectiveness as a gas barrier coating. Moreover, the high-water retention property demonstrated super-bubble-phobicity in water and inhibited gas bubble permeation. This ultrafast self-healing gas barrier coating has the potential to be used not only for food products, but also for electronic devices, pharmaceutical packaging, and gas separation applications by optimizing it for future applications. These potential applications suggest that the coating developed in this study will be a 
promising material that can play an extraordinary role in economic and social contributions toward waste reduction and the achievement of the SDGs.

\section{METHODS}

Materials. branched polyethyleneimine (bPEI, $\mathrm{M}_{\mathrm{w}} \sim 10,000$, refractive index 1.529, Polysciences, Inc., PA, US), polyacrylic acid (PAA, $\mathrm{M}_{\mathrm{w}} \sim 250,000$, refractive index 1.527, Wako Pure Chemical Industry Ltd., Osaka, Japan), $\mathrm{CaCl}_{2}$ (Wako Pure Chemical Industry Ltd., Osaka, Japan), and sodium montmorillonite nanoclay (MMT, refractive index 1.426, Fluorochem Ltd, Glossop, UK) were used for the preparation of self-healing nanoclay coatings. MMT can be dissociated in solution by using sodium montmorillonite, which also acts as an ion. Deionized water (Milli-Q Direct-Q UV3, Merck KGaA, Darmstadt, Germany) was used for the precursor solution and rinse for $\mathrm{LbL}$ self-assembly method. All polymers were used without further purification. Glass substrates $(26 \times 76 \mathrm{~mm}$, thickness $1.0 \mathrm{~mm}$, refractive index 1.52, S1111, Matsunami Glass Ind., Ltd., Kishiwada, Japan) and polyethylene film (PE, thickness $50 \mu \mathrm{m}$, TOCHISEN CHEMICAL INDUSTRY CO.,LTD., Tochigi, Japan) were used as substrates.

Preparation of self-healing coatings. Polycation and polyanion solution were composed of bPEI $4 \mathrm{mg} / \mathrm{mL}$ and PAA 4 $\mathrm{mg} / \mathrm{mL}$, respectively, with $10 \mathrm{mM} \mathrm{CaCl}_{2}$ and $\mathrm{MMT}$ at different concentration. To improve the dispersion of MMT, the solution was treated with an ultrasonic homogenizer for $1 \mathrm{~min}$. All solutions were stirred for $12 \mathrm{~h}$. The solution $\mathrm{pH}$ of polycation and polyanion were 10.5 and 3.1, respectively, and they were not further adjusted. All substrates were cleaned in $\mathrm{KOH}$ solution $\left(1: 120: 240 \mathrm{wt} \% \mathrm{KOH} / \mathrm{H}_{2} \mathrm{O} / \mathrm{EtOH}\right)$ for $5 \mathrm{~min}$ and then rinsed thoroughly with ultrapure water. The substrate was alternately immersed in polycation or polyanion solutions for $5 \mathrm{~min}$ and then rinsed with pure water after deposition of each layer for every 2 min to completely remove residual $\mathrm{CaCl}_{2}$ in the PEMs.

Characterization. The surface morphology, thickness, roughness, and self-healing performance of the coatings were analyzed using a 3D scanning laser microscope (VK-X100, KEYENCE Corp., Osaka, Japan). The water retention was measured using an electronic analytical scale and calculated from the contact area and height of $5-\mu \mathrm{L}$ water droplets using a contact angle meter (Drop Master 300K, Kyowa Interface Science Co., Ltd, Saitama, Japan). Images of static and dynamic super-bubble-phobicity under water were captured using a monochrome high-speed camera (Chronos 1.4, Kron Technologies Inc., BC, Canada). The chemical properties were measured using FT-IR (Nicolet iS50, Thermo Fisher Scientific Inc., MA, US). Transmittance measurements in the spectral range of 300-1500 nm were carried out using a UV-visible/NIR spectrophotometer (V-780, JASCO Corp, Tokyo, Japan). A photo of each coating on glass substrates was acquired using an action camera (GoPro Hero 8, GoPro, Inc., CA, US). OTR for oxygen permeability was measured via differential pressure method using a gas permeation analysis system (GTR-10XFKS, GTR TEC Corp., Kyoto, Japan) that complies with ISO 15105-1 and JIS K7126 and can analyze the gas transmission rate and permeability coefficient of gases under controlling relative humidity. The OTR measurement was conducted via an entrusted measurement by Kanagawa Institute of Industrial Science and Technology (KISTEC), Kanagawa, Japan.

\section{ASSOCIATED CONTENT Supporting Information}

FT-IR spectra of coatings without $\mathrm{CaCl}_{2}$ and MMT; Surface roughness profiles $(\mathrm{Sz})$; Depth analysis at the damage before and after self-repairing; Photo image of self-healing nanoclay coatings after OTR measurements at $0 \%$ relative humidity; Movie about super-bubble-phobic surface.

\section{AUTHOR INFORMATION}

\section{Corresponding Author}

*E-mail: kengo.manabe@aist.go.jp.

\section{Present Addresses}

Research Institute for Advanced Electronics and Photonics, National Institute of Advanced Industrial Science and Technology (AIST), Higashi 1-1-1, Tsukuba, Ibaraki, 305-8565, Japan

\section{Author Contributions}

K.M. conceived, designed, and performed the experiments, analyzed the data, and wrote the paper. M.K. and Y.N. contributed to the data analysis. All authors discussed the results and reviewed the manuscript. K.M. was responsible for all figures in the main text and the Supporting Information.

\section{Funding Sources}

JSPS KAKENHI Grant Number JP19K23501, JP20H02456, a research grant from TOYO INSTITUTE of FOOD TECHNOLOGY in 2019 .

Notes

The authors declare no competing financial interests.

\section{ACKNOWLEDGMENTS}

This work was supported by JSPS KAKENHI Grant Number JP19K23501, JP20H02456, and a research grant from TOYO INSTITUTE of FOOD TECHNOLOGY in 2019. OTR measurements were conducted by Kanagawa Institute of Industrial Science and Technology (KISTEC), Kanagawa, Japan.

\section{ABBREVIATIONS}

LbL, layer-by-layer; MMT, Montmorillonite; bPEI, branched polyethyleneimine; PAA, polyacrylic acid; OTR, oxygen transmission rate; SDGs, Sustainable Development Goals.

\section{REFERENCES}

(1) Thuy, V. T. T.; Hao, L. T.; Jeon, H.; Koo, J. M.; Park, J.; Lee, E. S.; Hwang, S. Y.; Choi, S.; Park, J.; Oh, D. X. Sustainable, Self-Cleaning, Transparent, and Moisture/oxygen-Barrier Coating Films for Food Packaging. Green Chem. 2021, 23 (7), 2658-2667. https://doi.org/10.1039/D0GC03647A.

(2) Maes, C.; Luyten, W.; Herremans, G.; Peeters, R.; Carleer, R.; Buntinx, M. Recent Updates on the Barrier Properties of Ethylene Vinyl Alcohol Copolymer (EVOH): A Review. Polym. Rev. 2018, 58 (2), 209-246. https://doi.org/10.1080/15583724.2017.1394323.

(3) Xu, H.; Feng, Z.-X.; Xie, L.; Hakkarainen, M. Graphene OxideDriven Design of Strong and Flexible Biopolymer Barrier Films: From 
Smart Crystallization Control to Affordable Engineering. ACS Sustainable Chem. Eng. 2016, 4 (1), 334-349. https://doi.org/10.1021/acssuschemeng.5b01273.

(4) Rovera, C.; Ghaani, M.; Farris, S. Nano-Inspired Oxygen Barrier Coatings for Food Packaging Applications: An Overview. Trends Food Sci. Technol. 2020, 97, 210-220. https://doi.org/10.1016/j.tifs.2020.01.024.

(5) Ruggeri, E.; Kim, D.; Cao, Y.; Farè, S.; De Nardo, L.; Marelli, B. A Multilayered Edible Coating to Extend Produce Shelf Life. ACS Sustainable Chem. Eng. 2020, 8 (38), 14312-14321. https://doi.org/10.1021/acssuschemeng.0c03365.

(6) Arora, A.; Padua, G. W. Review: Nanocomposites in Food Packaging. J. Food Sci. 2010, 75 (1), R43-R49. https://doi.org/10.1111/j.1750-3841.2009.01456.x.

(7) Goulas, A. E. Overall Migration from Commercial Coextruded Food Packaging Multilayer Films and Plastics Containers into Official EU Food Simulants. Eur. Food Res. Technol. 2001, 212 (5), 597-602. https://doi.org/10.1007/s002170000294.

(8) Gaikwad, K. K.; Singh, S.; Lee, Y. S. Oxygen Scavenging Films in Food Packaging. Environ. Chem. Lett. 2018, 16 (2), 523-538. https://doi.org/10.1007/s10311-018-0705-z.

(9) Mustafa, F.; Andreescu, S. Nanotechnology-Based Approaches for Food Sensing and Packaging Applications. RSC Adv. 2020, 10 (33), 19309-19336. https://doi.org/10.1039/D0RA01084G.

(10) Priolo, M. A.; Holder, K. M.; Guin, T.; Grunlan, J. C. Recent Advances in Gas Barrier Thin Films via Layer-by-Layer Assembly of Polymers and Platelets. Macromol. Rapid Commun. 2015, 36 (10), 866-879. https://doi.org/10.1002/marc.201500055.

(11) Kim, T.; Tran, T. H.; Hwang, S. Y.; Park, J.; Oh, D. X.; Kim, B.S. Crab-on-a-Tree: All Biorenewable, Optical and Radio Frequency Transparent Barrier Nanocoating for Food Packaging. ACS Nano. 2019 13 (4), 3796-3805. https://doi.org/10.1021/acsnano.8b08522.

(12) Wang, J.; Xu, X.; Zhang, J.; Chen, M.; Dong, S.; Han, J.; Wei, M. Moisture-Permeable, Humidity-Enhanced Gas Barrier Films Based on Organic/Inorganic Multilayers. ACS Appl. Mater. Interfaces 2018, 10 (33), 28130-28138. https://doi.org/10.1021/acsami.8b09740.

(13) H Tayeb, A.; Tajvidi, M. Sustainable Barrier System via SelfAssembly of Colloidal Montmorillonite and Cross-Linking Resins on Nanocellulose Interfaces. ACS Appl. Mater. Interfaces 2019, 11 (1), 1604-1615. https://doi.org/10.1021/acsami.8b16659.

(14) Yu, J.; Ruengkajorn, K.; Hare, D. O.; Chen, C.; Buffet, J.-C. High Gas Barrier Coating Using Non-Toxic Nanosheet Dispersions for Flexible Food Packaging Film. Nat. Commun. 2019, 10, 2398. https://doi.org/10.1038/s41467-019-10362-2.

(15) Sun, P. Z.; Yang, Q.; Kuang, W. J.; Stebunov, Y. V.; Xiong, W. Q.; Yu, J.; Nair, R. R.; Katsnelson, M. I.; Yuan, S. J.; Grigorieva, I. V.; Lozada-Hidalgo, M.; Wang, F. C.; Geim, A. K. Limits on Gas Impermeability of Graphene. Nature 2020, 579 (7798), 229-232. https://doi.org/10.1038/s41586-020-2070-x.

(16) Zahid, M.; Del Río Castillo, A. E.; Thorat, S. B.; Panda, J. K.; Bonaccorso, F.; Athanassiou, A. Graphene Morphology Effect on the Gas Barrier, Mechanical and Thermal Properties of Thermoplastic Polyurethane. Compos. Sci. Technol. 2020, 200, 108461. https://doi.org/10.1016/j.compscitech.2020.108461.

(17) Wang, J.; Gardner, D. J.; Stark, N. M.; Bousfield, D. W.; Tajvidi, M.; Cai, Z. Moisture and Oxygen Barrier Properties of Cellulose Nanomaterial-Based Films. ACS Sustainable Chem. Eng. 2018, 6 (1), 4970. https://doi.org/10.1021/acssuschemeng.7b03523.

(18) Chowdhury, R. A.; Nuruddin, M.; Clarkson, C.; Montes, F.; Howarter, J.; Youngblood, J. P. Cellulose Nanocrystal (CNC) Coatings with Controlled Anisotropy as High-Performance Gas Barrier Films. ACS Appl. Mater. Interfaces 2019, 11 (1), 1376-1383. https://doi.org/10.1021/acsami.8b16897.
(19) Priolo, M. A.; Gamboa, D.; Holder, K. M.; Grunlan, J. C. Super Gas Barrier of Transparent Polymer-Clay Multilayer Ultrathin Films. Nano Lett. 2010, $10 \quad$ (12), 4970-4974. https://doi.org/10.1021/nl103047k.

(20) Noh, M. J.; Oh, M. J.; Choi, J. H.; Yu, J. C.; Kim, W.-J.; Park, J.; Chang, Y.-W.; Yoo, P. J. Layer-by-Layer Assembled Multilayers of Charged Polyurethane and Graphene Oxide Platelets for Flexible and Stretchable Gas Barrier Films. Soft Matter 2018, 14 (32), 6708-6715. https://doi.org/10.1039/c8sm00706c.

(21) Yuan, W.; Weng, G.-M.; Lipton, J.; Li, C. M.; Van Tassel, P. R.; Taylor, A. D. Weak Polyelectrolyte-Based Multilayers via Layer-byLayer Assembly: Approaches, Properties, and Applications. Adv. Colloid Interface Sci. $\quad \mathbf{2 0 2 0}, \quad 282, \quad 102200$. https://doi.org/10.1016/j.cis.2020.102200.

(22) Manabe, K.; Nara, H. Construction of Stable Biological Albumin/heparin Multilayers for Elastic Coatings on Hydrophobic Antithrombogenic Artificial Blood Vessels. Tribol. Int. 2021, 156, 106843 https://doi.org/10.1016/j.triboint.2020.106843

(23) Zhao, S.; Caruso, F.; Dähne, L.; Decher, G.; G. De Geest, B.; Fan, J.; Feliu, N.; Gogotsi, Y.; T. Hammond, P.; C. Hersam, M.; Khademhosseini, A.; Kotov, N.; Leporatti, S.; Li, Y.; Lisdat, F.; M. Liz-Marzán, L.; Moya, S.; Mulvaney, P.; L. Rogach, A.; Roy, S.; G. Shchukin, D.; G. Skirtach, A.; M. Stevens, M.; B. Sukhorukov, G.; S. Weiss, P.; Yue, Z.; Zhu, D.; J. Parak, W. The Future of Layer-by-Layer Assembly: A Tribute to ACS Nano Associate Editor Helmuth Möhwald. ACS Nano 2019, 13 (6), 6151-6169. https://doi.org/10.1021/acsnano.9b03326.

(24) Chen, X.-C.; Ren, K.-F.; Zhang, J.-H.; Li, D.-D.; Zhao, E.; Zhao, Z. J.; Xu, Z.-K.; Ji, J. Humidity-Triggered Self-Healing of Microporous Polyelectrolyte Multilayer Coatings for Hydrophobic Drug Delivery. Adv. Funct. Mater. 2015, 25 (48), 7470-7477. https://doi.org/10.1002/adfm.201503258.

(25) Skorb, E. V.; Andreeva, D. V. Layer-by-Layer Approaches for Formation of Smart Self-Healing Materials. Polym. Chem. 2013, 4 (18), 4834-4845. https://doi.org/10.1039/c3py00088e.

(26) Wang, Z.; Scheres, L.; Xia, H.; Zuilhof, H. Developments and Challenges in Self-healing Antifouling Materials. Adv. Funct. Mater. 2020, 30 (26), 1908098. https://doi.org/10.1002/adfm.201908098.

(27) Hao, X.; Wang, W.; Yang, Z.; Yue, L.; Sun, H.; Wang, H.; Guo, Z.; Cheng, F.; Chen, S. pH Responsive Antifouling and Antibacterial Multilayer Films with Self-Healing Performance. Chem. Eng. J. 2019, 356, 130-141. https://doi.org/10.1016/j.cej.2018.08.181.

(28) Li, Y.; Li, Q.; Zhang, C.; Cai, P.; Bai, N.; Xu, X. Intelligent SelfHealing Superhydrophobic Modification of Cotton Fabrics via SurfaceInitiated ARGET ATRP of Styrene. Chem. Eng. J. 2017, 323, 134-142. https://doi.org/10.1016/j.cej.2017.04.080.

(29) Xue, C.-H.; Wu, Y.; Guo, X.-J.; Liu, B.-Y.; Wang, H.-D.; Jia, S.T. Superhydrophobic, Flame-Retardant and Conductive Cotton Fabrics via Layer-by-Layer Assembly of Carbon Nanotubes for Flexible Sensing Electronics. Cellulose 2020, 27 (6), 3455-3468. https://doi.org/10.1007/s10570-020-03013-z.

(30) Qiu, X.; Li, Z.; Li, X.; Zhang, Z. Flame Retardant Coatings Prepared Using Layer by Layer Assembly: A Review. Chem. Eng. J. 2018, 334, 108-122. https://doi.org/10.1016/j.cej.2017.09.194.

(31) Song, Y.; Meyers, K. P.; Gerringer, J.; Ramakrishnan, R. K.; Humood, M.; Qin, S.; Polycarpou, A. A.; Nazarenko, S.; Grunlan, J. C. Fast Self-Healing of Polyelectrolyte Multilayer Nanocoating and Restoration of Super Oxygen Barrier. Macromol. Rapid Commun. 2017, 38 (10), 1700064. https://doi.org/10.1002/marc.201700064.

(32) Liang, B.; Zhong, Z.; Jia, E.; Zhang, G.; Su, Z. Transparent and Scratch-Resistant Antifogging Coatings with Rapid Self-Healing Capability. ACS Appl. Mater. Interfaces 2019, acsami.9b09610. https://doi.org/10.1021/acsami.9b09610. 
(33) Pena-Francesch, A.; Jung, H.; Demirel, M. C.; Sitti, M. Biosynthetic Self-Healing Materials for Soft Machines. Nat. Mater. 2020, 19 (11), 1230-1235. https://doi.org/10.1038/s41563-020-0736-2.

(34) Jung, H.; Pena-Francesch, A.; Saadat, A.; Sebastian, A.; Kim, D. H.; Hamilton, R. F.; Albert, I.; Allen, B. D.; Demirel, M. C. Molecular Tandem Repeat Strategy for Elucidating Mechanical Properties of High-Strength Proteins. Proc. Natl. Acad. Sci. U. S. A. 2016, 113 (23), 6478-6483. https://doi.org/10.1073/pnas.1521645113.

(35) Pena-Francesch, A.; Jung, H.; Segad, M.; Colby, R. H.; Allen, B. D.; Demirel, M. C. Mechanical Properties of Tandem-Repeat Proteins Are Governed by Network Defects. ACS Biomaterials Science and Engineering 2018, 4 (3), 884-891. https://doi.org/10.1021/acsbiomaterials. $7 \mathrm{~b} 00830$.

(36) Yoo, D.; Shiratori, S. S.; Rubner, M. F. Controlling Bilayer Composition and Surface Wettability of Sequentially Adsorbed Multilayers of Weak Polyelectrolytes. Macromolecules 1998, 31 (13), 4309-4318. https://doi.org/10.1021/ma9800360.

(37) Shiratori, S. S.; Rubner, M. F. pH-Dependent Thickness Behavior of Sequentially Adsorbed Layers of Weak Polyelectrolytes. Macromolecules $\quad 2000, \quad 33 \quad$ (11), $4213-4219$. https://doi.org/10.1021/ma991645q.

(38) Manabe, K.; Tanaka, C.; Moriyama, Y.; Tenjimbayashi, M.; Nakamura, C.; Tokura, Y.; Matsubayashi, T.; Kyung, K.; Shiratori, S. Chitin Nanofibers Extracted from Crab Shells in Broadband Visible Antireflection Coatings with Controlling Layer-by-Layer Deposition and the Application for Durable Antifog Surfaces. ACS Appl. Mater. Interfaces $\quad$ 2016, $\quad 8 \quad$ (46), $31951-31958$. https://doi.org/10.1021/acsami.6b11786.

(39) Manabe, K. Growth of Porous Chitin-Nanofibrous Structure via Layer-by-Layer Self-Assembly under Existing Ionic Effects for Antireflective and Antifogging Coatings. Prog. Org. Coat. 2020, 142, 105599. https://doi.org/10.1016/j.porgcoat.2020.105599.

(40) Qin, S.; Song, Y.; Floto, M. E.; Grunlan, J. C. Combined High Stretchability and Gas Barrier in Hydrogen-Bonded Multilayer Nanobrick Wall Thin Films. ACS Appl. Mater. Interfaces 2017, 9 (9), 7903 7907. https://doi.org/10.1021/acsami.7b00844.

(41) Deng, Y.; Wang, T.; Guo, Y.; Qiu, X.; Qian, Y. Layer-by-Layer Self-Assembled Films of a Lignin-Based Polymer through Hydrogen
Bonding. ACS Sustainable Chem. Eng. 2015, 3 (6), 1215-1220. https://doi.org/10.1021/acssuschemeng.5b00178.

(42) Xu, G.; Liu, P.; Pranantyo, D.; Neoh, K.-G.; Kang, E.-T. Dextran- and Chitosan-Based Antifouling, Antimicrobial Adhesion, and Self-Polishing Multilayer Coatings from $\mathrm{pH}$-Responsive Linkages-Enabled Layer-by-Layer Assembly. ACS Sustainable Chem. Eng. 2018, 6 (3), 3916-3926. https://doi.org/10.1021/acssuschemeng.7b04286.

(43) Schoeler, B.; Kumaraswamy, G.; Caruso, F. Investigation of the Influence of Polyelectrolyte Charge Density on the Growth of Multilayer Thin Films Prepared by the Layer-by-Layer Technique. Macromolecules 2002, 35 (3), 889-897. https://doi.org/10.1021/ma011349p.

(44) Yang, Y.-H.; Malek, F. A.; Grunlan, J. C. Influence of Deposition Time on Layer-by-Layer Growth of Clay-Based Thin Films. Ind. Eng. Chem. Res. 2010, 49 (18), 8501-8509. https://doi.org/10.1021/ie100499x.

(45) Li, C.; Gu, Y.; Zacharia, N. S. Tuning Wet Adhesion of Weak Polyelectrolyte Multilayers. ACS Appl. Mater. Interfaces 2018, 10 (8), 7401-7412. https://doi.org/10.1021/acsami.7b18910.

(46) Gu, Y.; Weinheimer, E. K.; Ji, X.; Wiener, C. G.; Zacharia, N. S. Response of Swelling Behavior of Weak Branched Poly(ethylene imine)/Poly(acrylic Acid) Polyelectrolyte Multilayers to Thermal Treatment. Langmuir 2016, 32 (24), 6020-6027. https://doi.org/10.1021/acs.langmuir.6b00206.

(47) Manabe, K.; Matsubayashi, T.; Tenjimbayashi, M.; Moriya, T.; Tsuge, Y.; Kyung, K. H.; Shiratori, S. Controllable Broadband Optical Transparency and Wettability Switching of Temperature-Activated Solid/Liquid-Infused Nanofibrous Membranes. ACS Nano 2016, 10 (10), 9387-9396. https://doi.org/10.1021/acsnano.6b04333.

(48) Manabe, K.; Nishizawa, S.; Kyung, K. H.; Shiratori, S. Optical Phenomena and Antifrosting Property on Biomimetics Slippery FluidInfused Antireflective Films via Layer-by-Layer Comparison with Superhydrophobic and Antireflective Films. ACS Appl. Mater. Interfaces 2014, 6 (16), 13985-13993. https://doi.org/10.1021/am503352x.

(49) Wang, X.; Liu, F.; Zheng, X.; Sun, J. Water-enabled Self-healing of Polyelectrolyte Multilayer Coatings. Angew. Chem. Int. Ed. 2011, 50 (48), 11378-11381. https://doi.org/10.1002/anie.201105822.

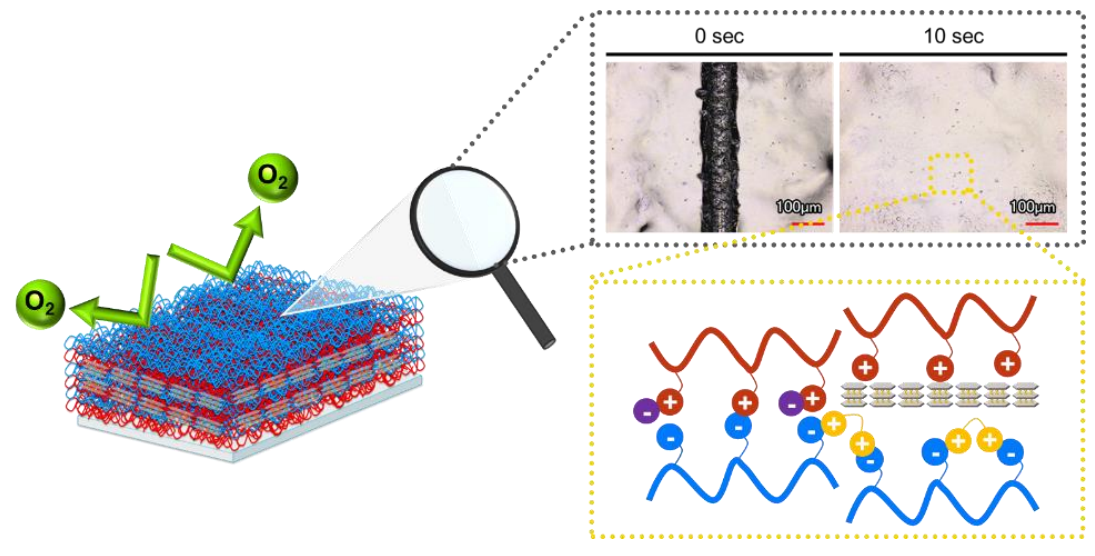

Table of Contents artwork 